\title{
Research Article \\ Optimal Design of a Centrifugal Compressor Impeller Using Evolutionary Algorithms
}

\author{
Soo-Yong Cho, ${ }^{1}$ Kook-Young Ahn, ${ }^{2}$ \\ Young-Duk Lee, ${ }^{2}$ and Young-Cheol Kim ${ }^{2}$ \\ ${ }^{1}$ Department of Mechanical and Aerospace Engineering (RECAPT), Gyeongsang National University, \\ 900 Gajwa-dong, Gyeongnam, Jinju 660-701, Republic of Korea \\ 2 Department of Eco-Machinery, Korea Institute of Machinery and Materials, 171 Jang-dong, \\ Daejeon 305-343, Republic of Korea
}

Correspondence should be addressed to Soo-Yong Cho, sycho@gnu.ac.kr

Received 29 May 2012; Revised 7 September 2012; Accepted 7 September 2012

Academic Editor: Gerhard-Wilhelm Weber

Copyright (C) 2012 Soo-Yong Cho et al. This is an open access article distributed under the Creative Commons Attribution License, which permits unrestricted use, distribution, and reproduction in any medium, provided the original work is properly cited.

\begin{abstract}
An optimization study was conducted on a centrifugal compressor. Eight design variables were chosen from the control points for the Bezier curves which widely influenced the geometric variation; four design variables were selected to optimize the flow passage between the hub and the shroud, and other four design variables were used to improve the performance of the impeller blade. As an optimization algorithm, an artificial neural network (ANN) was adopted. Initially, the design of experiments was applied to set up the initial data space of the ANN, which was improved during the optimization process using a genetic algorithm. If a result of the ANN reached a higher level, that result was re-calculated by computational fluid dynamics (CFD) and was applied to develop a new ANN. The prediction difference between the ANN and CFD was consequently less than $1 \%$ after the 6 th generation. Using this optimization technique, the computational time for the optimization was greatly reduced and the accuracy of the optimization algorithm was increased. The efficiency was improved by $1.4 \%$ without losing the pressure ratio, and Pareto-optimal solutions of the efficiency versus the pressure ratio were obtained through the 21st generation.
\end{abstract}

\section{Introduction}

Centrifugal compressors are still used in small engines of commuter aircraft due to their high compressor ratio and narrow installation space compared to axial-type compressors. In industrial fields, centrifugal compressors are also still widely used. Most turbochargers in vehicles adopt a centrifugal-type compressor. The efficiency of a centrifugal compressor may be $3-4 \%$ less than that of an axial-type compressor under the same operating conditions. Nonetheless, centrifugal compressors have several advantages. For instance, they are less sensitive to changes in the mass flowrate, have low manufacturing cost, and are easy to assemble. 
The efficiency or the pressure ratio of the centrifugal compressor greatly depends on the shape of the impeller and the flow passage between the hub and the shroud. For the design of the flow passage and the impeller blade, several methods [1-6] have been suggested. However, the configuration of the compressor is determined by the designer's experience and intuition. Thus, an initially designed compressor can be modified to improve its performance based on the flow structures within the passage after calculations using computational fluid dynamics (CFD).

To design a compressor systematically while reducing the influence by designers, design techniques using optimization methods [7-11] have been developed on the basis of the design variables that can control the shape of the compressor. However, the superiority of these design techniques could not be validated in experimental results due to the difficulties in properly conducting experiments. In particular, performance tests of a high-speed compressor are limited by the constraint that such tests require a torque meter that can operate at a high rotational speed. Additionally, insulation of the test facility greatly affects the amount of experimental uncertainty in measurement of the isentropic efficiency.

In this study, an artificial neural network (ANN) algorithm was adopted as a systematic design technique. Initially, the ANN was learned and trained based on input data selected using the design of experiments (DOE). The ANN was operated with a genetic algorithm (GA) in order to improve its accuracy during an optimization process; therefore, the computational time was reduced and a globally optimized result was obtained. This design technique with the ANN was applied to the compressor of an Eckardt impeller $[12,13]$ for comparison of experimental results and a validation of this optimization method.

In general, the shape of a compressor is expressed using a wide range of point data. However, these data cannot be applied to design variables directly. To select appropriate design variables, the shape of the compressor should be reexpressed in terms of an analytic function from the data of numerous points. Therefore, this study shows that any compressor, even those used in industrial fields, can be optimized using a fast and accurate optimization algorithm.

\section{Redesign of the Eckardt Impeller}

\subsection{Shape Parameters}

Flow passage of a centrifugal compressor is expressed on the meridian plane. The shape of a three-dimensional impeller is illustrated by the wrap angle $(\theta)$ and the radius, as shown in Figure 1. Profiles of the hub and shroud on the meridian plane can determine the flow passage, and the infinitesimal length $(\mathrm{dm})$ of the meridional curve with the radius of $r_{\text {ref }}$ can be expressed as (2.1). At that location, the blade angle $(\beta)$ of the impeller can be obtained using the wrap angle:

$$
\begin{gathered}
d m=\sqrt{(d z)^{2}+(d r)^{2}}, \\
\beta=\arctan \left(\frac{r_{\mathrm{ref}} d \theta}{d m}\right) .
\end{gathered}
$$

The shape of the impeller is usually expressed via point data along the blade on the suction surface and the pressure surface at the hub and tip. Figure 2 shows the point data of the Eckardt A-type impeller [14], which was chosen as a reference centrifugal compressor 


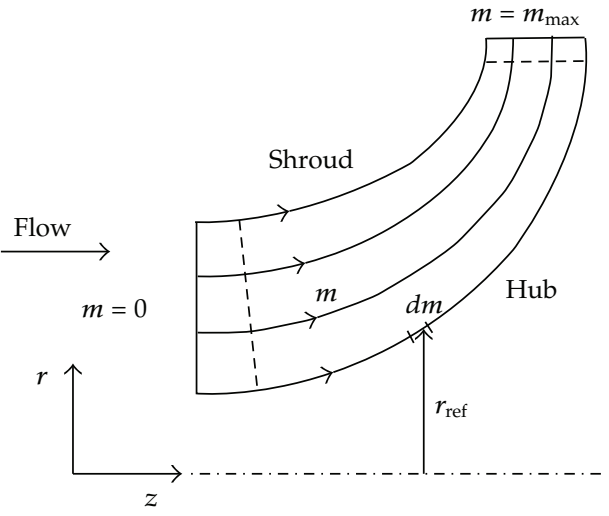

(a)

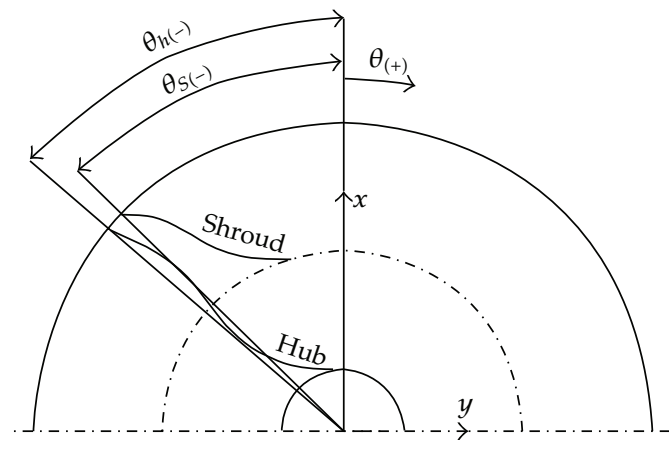

(b)

Figure 1: Definition of the geometry to illustrate a centrifugal compressor impeller: (a) meridian plane, and (b) wrap angle and Cartesian coordinates.

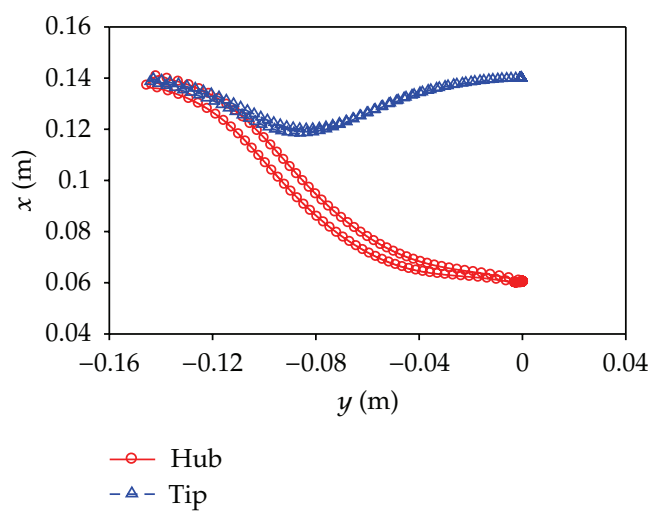

(a)

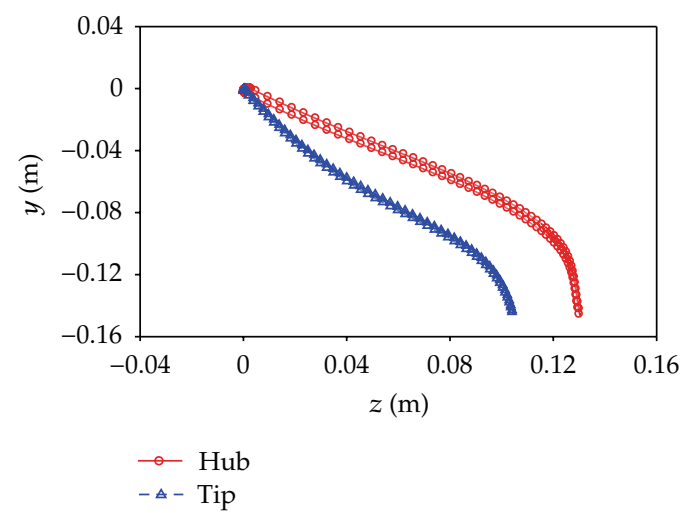

(b)

Figure 2: Impeller shape as expressed in Cartesian coordinates: (a) on the $x-y$ plane and (b) on the $y$-z plane.

for application of the optimization technique, because the performance and flow structures of this impeller were available. This large set of point data cannot be applied to design variables, therefore, the blade profile should be expressed by an analytic function. Initially, camber lines and the thickness of the blade were calculated at the hub and tip from the point data. These values are shown in Figure 3. In addition, the point data for the flow passage at the hub and shroud are shown in Figure 4.

\subsection{Shape Curve}

The shape expressed by the point data should be converted to an analytic function so as to choose the proper design variables for optimization. In this study, the Bezier curve was adopted as this curve is superior in terms of smoothness and continuity:

$$
R(u)=\sum_{i=0}^{n} B_{n, i}(u) V_{i}
$$




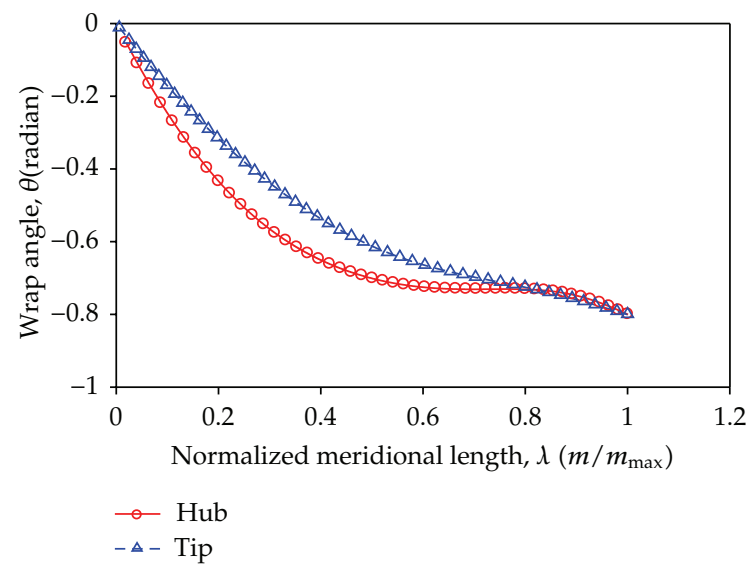

(a)

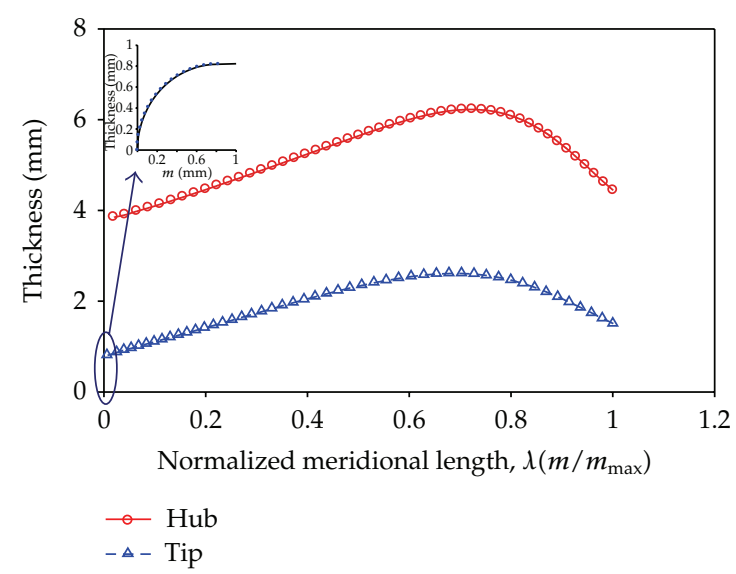

(b)

Figure 3: Profile of (a) wrap angle and (b) thickness along the camber line of the blade.

where $B_{n, i}(u)$ is the Bezier coefficient, $u$ is the independent variable, and $V_{i}$ is the control point. From the given point data, the control points of the Bezier curve can be calculated using the matrix of $[R]=[B][V]$ from (2.3). In this case, additional point data beyond the control points can be applied, and the control points can be obtained by $[V]=\left\{[B]^{t}[B]^{-1}\right\}[B]^{t}[R]$. However, the slope can change at the ends of the curve depending on the applied point data. Therefore, a boundary condition of the slope was added at the inlet and the exit to connect it smoothly to the other parts.

$$
\left[\begin{array}{c}
R_{k=1} \\
R_{k=2} \\
\vdots \\
R_{u=0}^{\prime} \\
R_{u=1}^{\prime}
\end{array}\right]=\left[\begin{array}{c}
B_{n, i}\left(u_{k=1}\right) \\
B_{n, i}\left(u_{k=2}\right) \\
\vdots \\
n\left(B_{n-1, i-1}(0)-B_{n, 1}(0)\right) \\
n\left(B_{n-1, i-1}(1)-B_{n, 1}(1)\right)
\end{array}\right]\left[\begin{array}{c}
V_{i=0} \\
\vdots \\
V_{i=n}
\end{array}\right]
$$


Table 1: Control points for the flow passage and design variables.

\begin{tabular}{lccccc}
\hline & At the shroud & & & At the hub & \\
Points & $z / R_{2}$ & $r / R_{2}$ & Points & $z / R_{2}$ & $r / R_{2}$ \\
\hline Ps1 & 0.0 & 0.700 & Ph1 & 0.0 & 0.300 \\
Ps2 & 0.078 & 0.695 & Ph2 & 0.223 & 0.350 \\
Ps3 & 0.184 & $0.733(\phi 1)$ & $\mathrm{Ph} 3$ & 0.394 & $0.400(\phi 3)$ \\
Ps4 & $0.399(\phi 2)$ & 0.587 & $\mathrm{Ph} 4$ & $0.629(\phi 4)$ & 0.581 \\
Ps5 & 0.496 & 0.886 & $\mathrm{Ph} 5$ & 0.632 & 0.801 \\
Ps6 & 0.520 & 1.0 & $\mathrm{Ph} 6$ & 0.650 & 1.0 \\
\hline
\end{tabular}

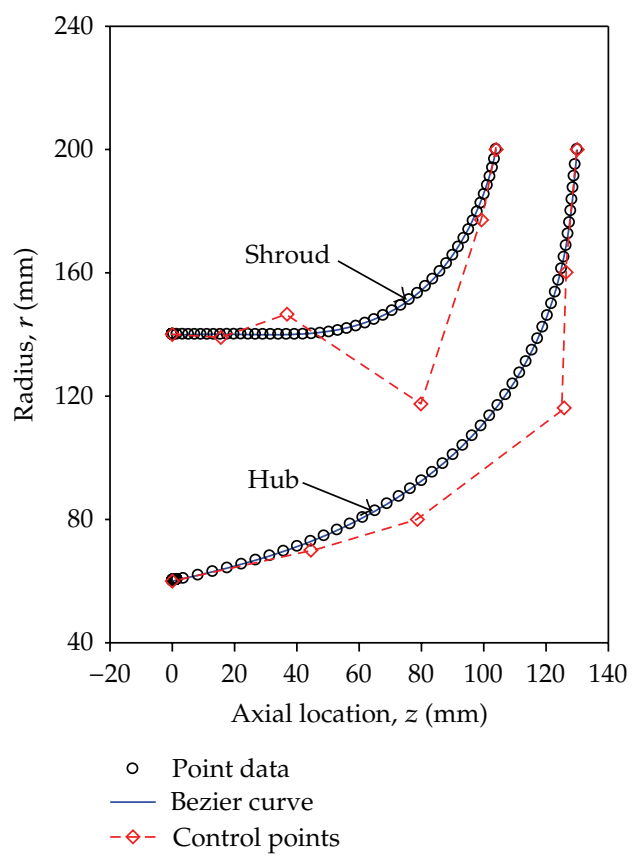

Figure 4: Comparison of the Bezier curves and point data for the flow passage.

where the subscript $k$ refers to the order of the points data. Control points were calculated using a transpose matrix and an inverse matrix of the Bezier coefficients.

The flow passage was redesigned using a Bezier curve formulated by six control points at the hub and shroud, as shown in Figure 4 . The Bezier curves agreed precisely with the point data despite that only six control points were applied at each line. However, more control points are needed if the curve becomes uneven. For the impeller blade angle calculated using (2.2) from the wrap angle of the blade, the difference between the point data and the Bezier curves is shown in Figure 5 with different numbers of control points. The Bezier curve at the hub was in good agreement with the point data when eight control points were applied. The application of additional control points in excess of eight did not lead to any improvements. At the tip, seven control points showed good agreement. The values of the control points at each line are recorded as normalized values, with the exception of the blade angle, in Tables 1 and 2 . 
Table 2: Control points for the blade angle and design variables.

\begin{tabular}{lccccc}
\hline & At the tip & & \multicolumn{3}{c}{ At the hub } \\
Points & $\lambda$ & $\beta$ [angle] & Points & $\lambda$ & $\beta$ [angle] \\
\hline Pt1 & 0.0 & -61.845 & $\mathrm{~Pb} 1$ & 0.0 & -36.399 \\
Pt2 & 0.167 & -59.627 & $\mathrm{~Pb} 2$ & 0.143 & -27.859 \\
Pt3 & 0.335 & $-30.022(\phi 5)$ & $\mathrm{Pb} 3$ & 0.286 & $-39.754(\phi 7)$ \\
Pt4 & 0.499 & -84.158 & $\mathrm{~Pb} 4$ & 0.429 & 36.429 \\
Pt5 & 0.661 & $46.949(\phi 6)$ & $\mathrm{Pb} 5$ & 0.527 & -82.025 \\
Pt6 & 0.833 & -41.912 & $\mathrm{~Pb} 6$ & 0.714 & $80.409(\phi 8)$ \\
Pt7 & 1.0 & -30.853 & $\mathrm{~Pb} 7$ & 0.857 & -26.313 \\
& - & - & $\mathrm{Pb} 8$ & 1.0 & -30.514 \\
\hline
\end{tabular}

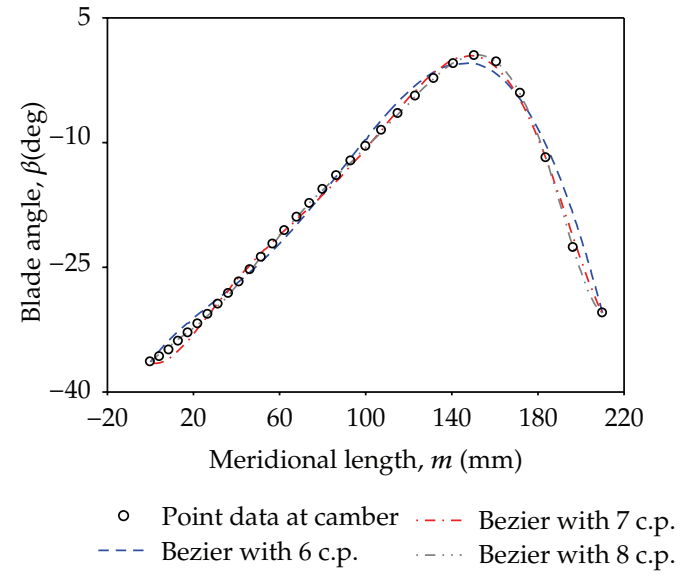

(a)

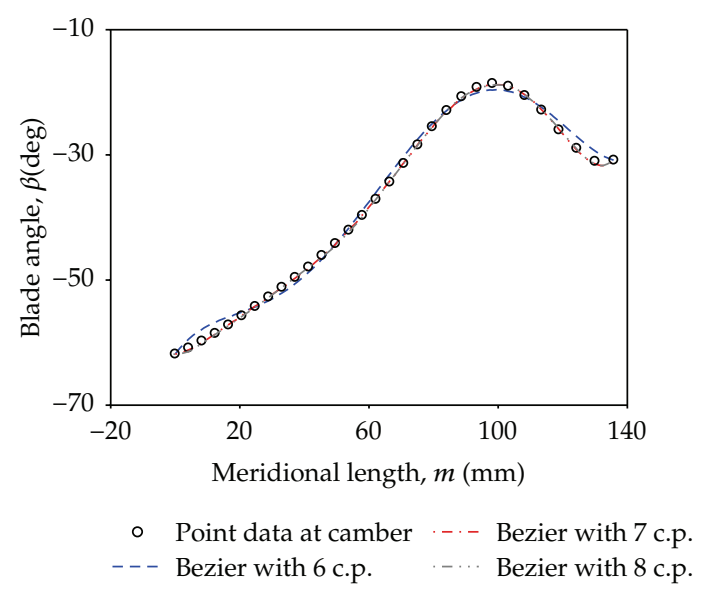

(b)

Figure 5: Comparison of the Bezier curves for the number of control points at the (a) hub and the (b) tip.

\subsection{Numerical Analysis}

To calculate flow structures of a three-dimensional compressible turbulent flow, CFX-12 [15] was used. A high resolution, which offers greater accuracy than the second-order finite difference, was applied for discretization, and the $k-\varepsilon$ turbulence model was adopted. From a test of turbulence models, the $k-\varepsilon$ model predicted the flow structure well within the passage of the rotating machinery. The computational domains were separated into the inlet domain, the impeller domain, and the diffuser domain, as the impeller was installed between the inlet and the diffuser. The interfaces between each domain were connected via the frozen rotor method.

For the boundary conditions, total pressure was applied at the inlet with medium turbulence intensity (5\%), and the mass flowrate was used at the outlet. A periodic boundary condition was applied to the surface in the circumferential direction, and a wall boundary was used on the hub and the shroud. Tip clearance was inserted between the impeller tip and the casing. In a test of grid independency, the computed results were similar when more than 150,000 elements were applied in the computational domain. In this computation, more than 220,000 elements were used in the computational domain, and the first grid away 


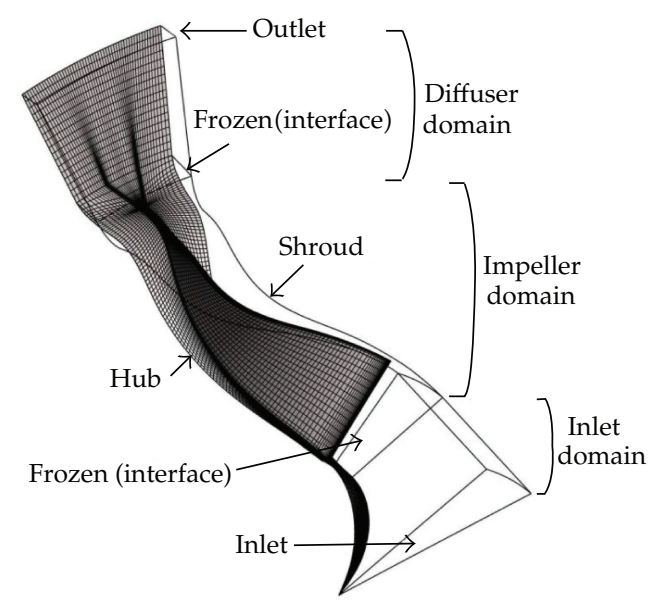

Figure 6: Computational grids and domains with boundaries.

from the wall was less than 5 for $y+$. Thirty-five meshes were applied along the spanwise direction including seven meshes for tip clearance modeling. An O-type grid of six meshes was applied around the blade to improve the grid orthogonality near the wall. The grids in the computational domain are shown in Figure 6. Convergence was achieved when all residuals (RMS) were reduced by at least 10 to the sixth order in magnitude.

Computational results at the design point showed total-to-total efficiency of $91.9 \%$ and a pressure ratio of 1.89 regardless of whether the calculation was conducted on the computational domain constructed using the point data or the Bezier curves. In a previous experiment [14], the total-to-total efficiency and the pressure ratio were obtained as $88.9 \%$ and 1.91 , respectively. Although the efficiency was overestimated by various factors in the computation, the overestimated efficiency and the underestimated pressure ratio were predicted consistently in the comparison with the experimental results at the off-design points.

Figure 7 shows the relative velocities at the center plane between blade and blade. The computed velocities were compared to those measured along the lines aligned normal to the meridional shroud contour [16]. Two-computed results were very similar. This indicates that the point data were converted well to the analytic function of the Bezier curve. However, these computational results show some discrepancy from the experimental data. In particular, this discrepancy is increased near the shroud when the flow approaches the exit of the impeller. The characteristics of this flow structure suggest that the tip clearance near the exit in the actual model would be infinitesimally smaller than that in the computational model: hence, this different tip clearance causes underestimation of the pressure ratio in CFD.

\section{Design Variables and Objective Function}

\subsection{Effect of Control Points}

Although the point data for the compressor configuration were converted to four Bezier curves; two for the flow passage at the hub and shroud, and the other two for the impeller blade at the shroud and tip, it is not possible to apply all of the control points of the Bezier curves to the design variables, because there are too many. Moreover, some of them are 


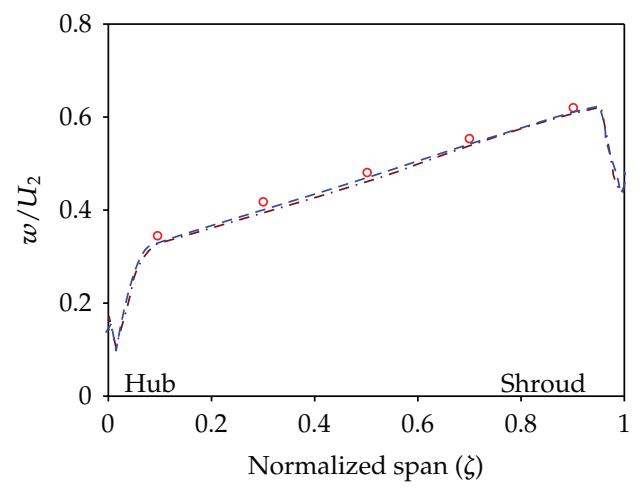

$\begin{array}{rll}\circ \quad & \text { Experiment } \\ --- & \text { Comp. (point data) }\end{array}$

(a)

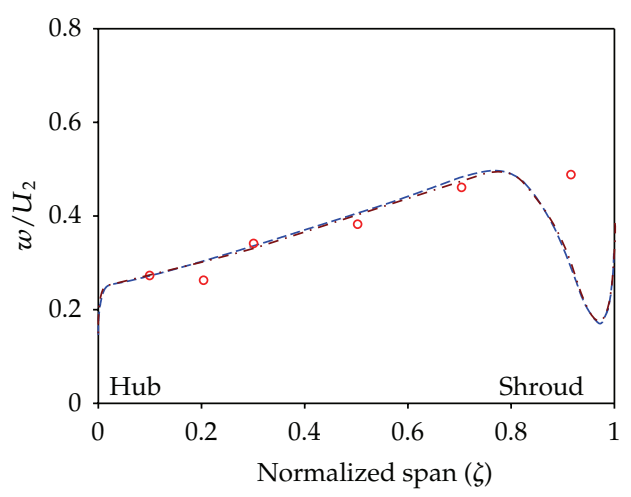

- Experiment - - Comp. (point data)

(c)

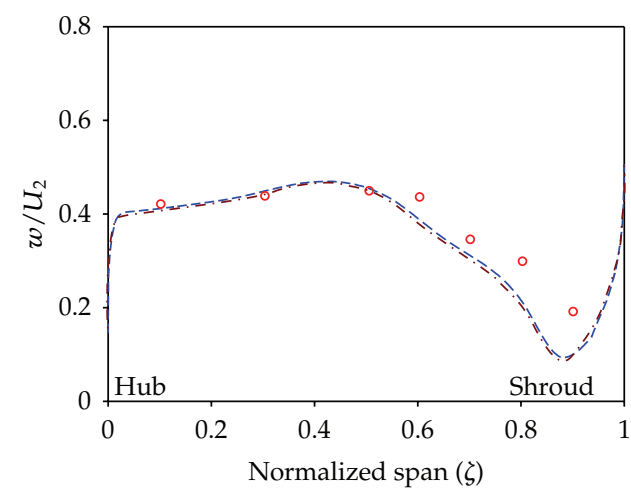

$\begin{array}{ll}\circ \quad \text { Experiment } \\ - & \text { Comp. (point data) }\end{array}$

(e)

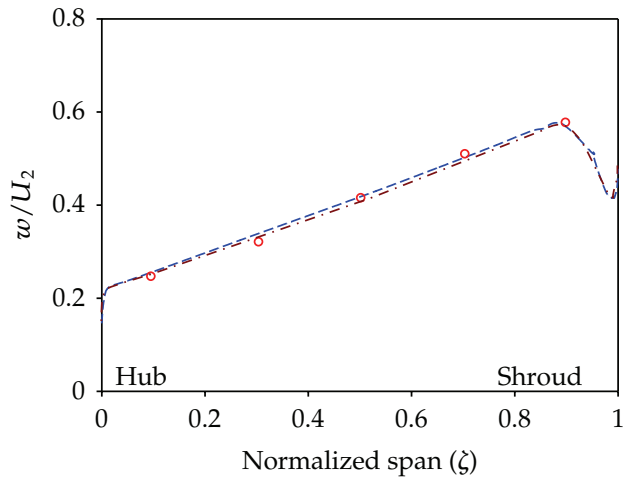

- Experiment

- - Comp. (point data)

.. Comp. (Bezier)

(b)

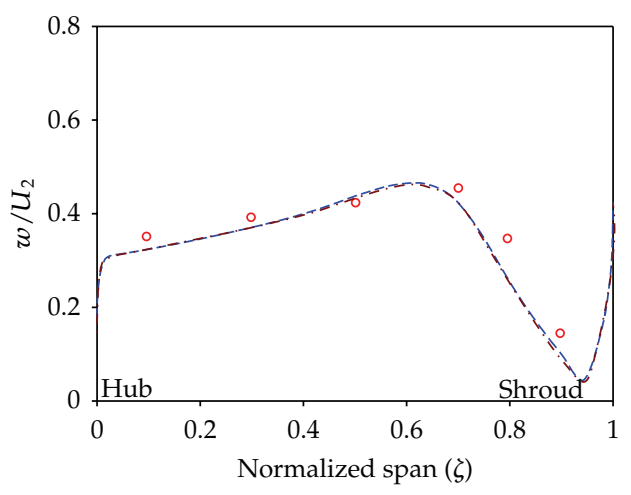

- Experiment $\quad . \cdot-$ Comp. (Bezier) - - Comp. (point data)

(d)

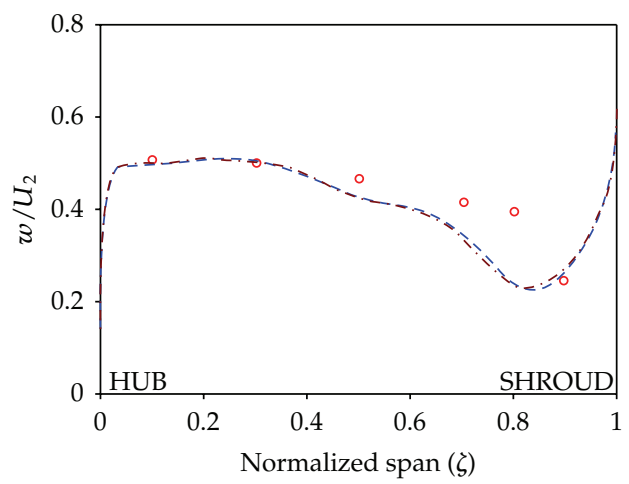

$\begin{array}{ll}\circ & \text { Experiment } \\ --- & \text { Comp. (point data) }\end{array}$

-..- Comp. (Bezier)

(f)

Figure 7: Relative velocities at half pitch along the spanwise direction: (a) $\lambda=0.08$, (b) $\lambda=0.43$, (c) $\lambda=0.59$, (d) $\lambda=0.73$, (e) $\lambda=0.87$, and (f) $\lambda=1.01$. 


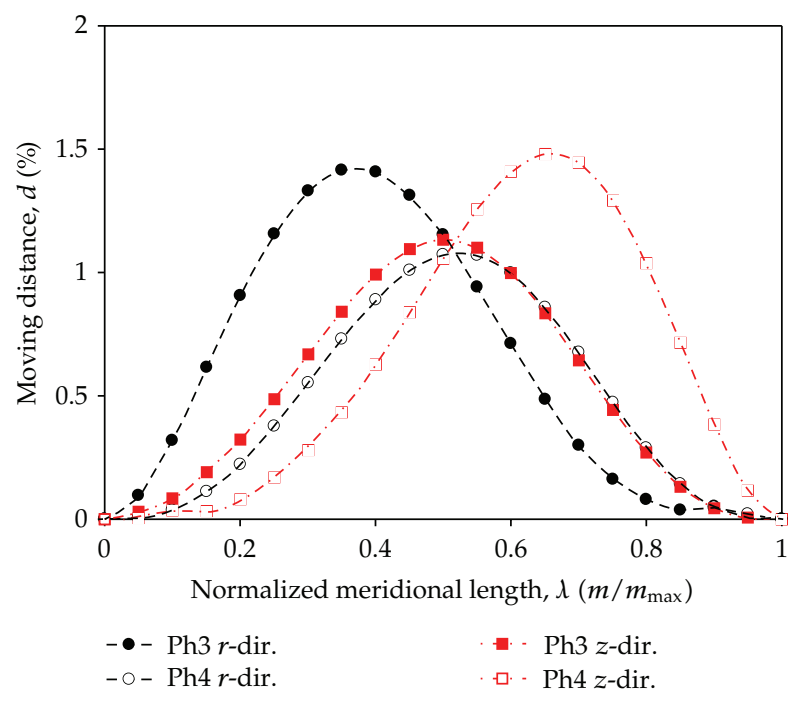

Figure 8: Hub contour change versus control points moving at the hub of the flow passage.

redundant regarding shape changes. Hence, control points that have a strong effect on the performance should be chosen to be used as design variables. As the performance of the compressor is closely related to the shape change, all control points are evaluated with respect to their effect on the compressor configuration.

Figure 8 shows the hub contour change of the flow passage when control points Ph3 and $\mathrm{Ph} 4$ are moved equally by a distance of $5 \%$ of the impeller exit radius $\left(R_{2}\right)$ to the $r$ direction and $z$-direction, respectively. Other control points are fixed so as not to change the slope at the inlet and exit of the impeller. In the Ph3 case, moving to the $r$-direction is more sensitive in terms of the contour change than moving to the $z$-direction. Conversely, moving to the $z$-direction is more sensitive for the $\mathrm{Ph} 4$ control point. Therefore, these $\mathrm{Ph} 3$ and $\mathrm{Ph} 4$ control points depending on the moving direction can be adopted as design variables, because these points fully express the change of the hub contour. After applying the same method to the shroud contour, moving to the $r$-direction of Ps 3 and moving to the $z$-direction of Ps4 sufficiently accounted for the change of the shroud contour.

The effects of the control points for the blade angle contour are compared in Figure 9. In the Bezier curve for the blade angle contour at the hub, eight control points were chosen. Among them, two control points at the inlet and the exit were not moved in order to fix the blade inlet and exit angle, because the compressor was operated at the same rotational speed. When the four control points of Pb3-Pb6 were equally moved by ten degrees, the effect on the blade angle contour was similar at each variable. When $\mathrm{Pb} 3$ and $\mathrm{Pb} 6$ were chosen as the design variables, the contour change of the blade angle at the hub could be expressed without using $\mathrm{Pb} 4$ and $\mathrm{Pb} 5$. At the impeller tip, the contour change of the blade angle was fully displaced with the use of only two control points, Pt3 and Pt5.

\subsection{Design Variables}

Eight design variables to optimize the Eckardt A-type compressor were chosen by examining the effect of each control point for the Bezier curve; four design variables were chosen for 


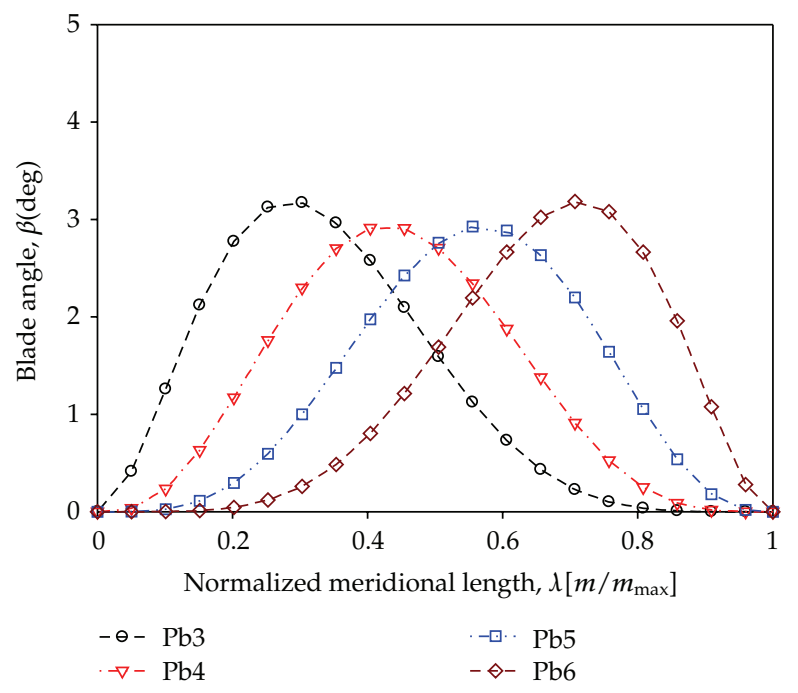

Figure 9: Blade angle contour change versus control points moving at the hub of the impeller.

the flow passage and other four were used for the impeller blade. Figure 10 shows the control points and the four design variables used to optimize the flow passage. Note that, Ph3 and Ps3 are limited in that they can move only along the $r$-direction, whereas Ph4 and Ps4 are limited to moving only along the $z$-direction. The locations of these variables are denoted as $\phi 1-\phi 4$ in Table 1. Figure 11 shows that the contour of the passage is fully controlled by the adopted design variables at the hub and shroud.

For the contour change of the blade angle, the control points $\mathrm{Pt} 3$ and $\mathrm{Pt} 5$ were adopted as the design variables at the tip, while $\mathrm{Pb} 3$ and $\mathrm{Pb} 6$ were used at the hub. Figure 12 shows the control points and design variables used to optimize the impeller blade, while Table 2 shows their locations, marked as $\phi 5-\phi 8$. These eight design variables are changed simultaneously during the optimization process, because the performance of the compressor is influenced not only by the passage shape but also by the impeller blade configuration. However, the configuration of the compressor could be altered significantly when the values of these design variables are changed in a wide region on the basis of the initial value. Hence, the range of the design variables was limited in terms of the upper and lower bound to avoid an inapplicable configuration. These limitations were obtained from the compressor configuration displayed according to the movement of the design variables. Table 3 shows the upper and lower bounds of the design variables. In particular, the lower bound of the design variable $\phi_{1}$, which is Ps3 pertaining to the passage contour at the shroud, is limited to the initial value for assembling the impeller within the casing.

\subsection{Objective Function}

An objective function for optimizing a compressor can be determined according to the users' requirements or by the application. For instance, it could be set for aerodynamic efficiency, a high pressure ratio, light weight, or small size. In this study, the efficiency and the pressure ratio are considered together as the objective function as follows:

$$
\mathrm{OF}=\omega_{\eta} P_{\eta}+\omega_{\Pi} P_{\Pi}
$$


Table 3: Upper and lower bounds of the design variables.

\begin{tabular}{lccc}
\hline Design variables & Minimum & Initial & Maximum \\
\hline$\phi 1, \operatorname{Ps} 3\left[r / R_{2}\right]$ & 0.733 & 0.733 & 0.808 \\
$\phi 2, \mathrm{Ps} 4\left[z / R_{2}\right]$ & 0.224 & 0.399 & 0.494 \\
$\phi 3, \mathrm{Ph} 3\left[r / R_{2}\right]$ & 0.275 & 0.400 & 0.525 \\
$\phi 4, \mathrm{Ph} 4\left[z / R_{2}\right]$ & 0.504 & 0.629 & 0.679 \\
$\phi 5, \mathrm{Pt} 3[$ angle] & -40.0 & -30.022 & -5.0 \\
$\phi 6, \mathrm{Pt} 5$ [angle] & 15.0 & 46.949 & 70.0 \\
$\phi 7, \mathrm{~Pb} 3$ [angle] & -50.0 & -39.754 & -28.0 \\
$\phi 8, \mathrm{~Pb} 6$ [angle] & -50.0 & 80.409 & 90.0 \\
\hline
\end{tabular}

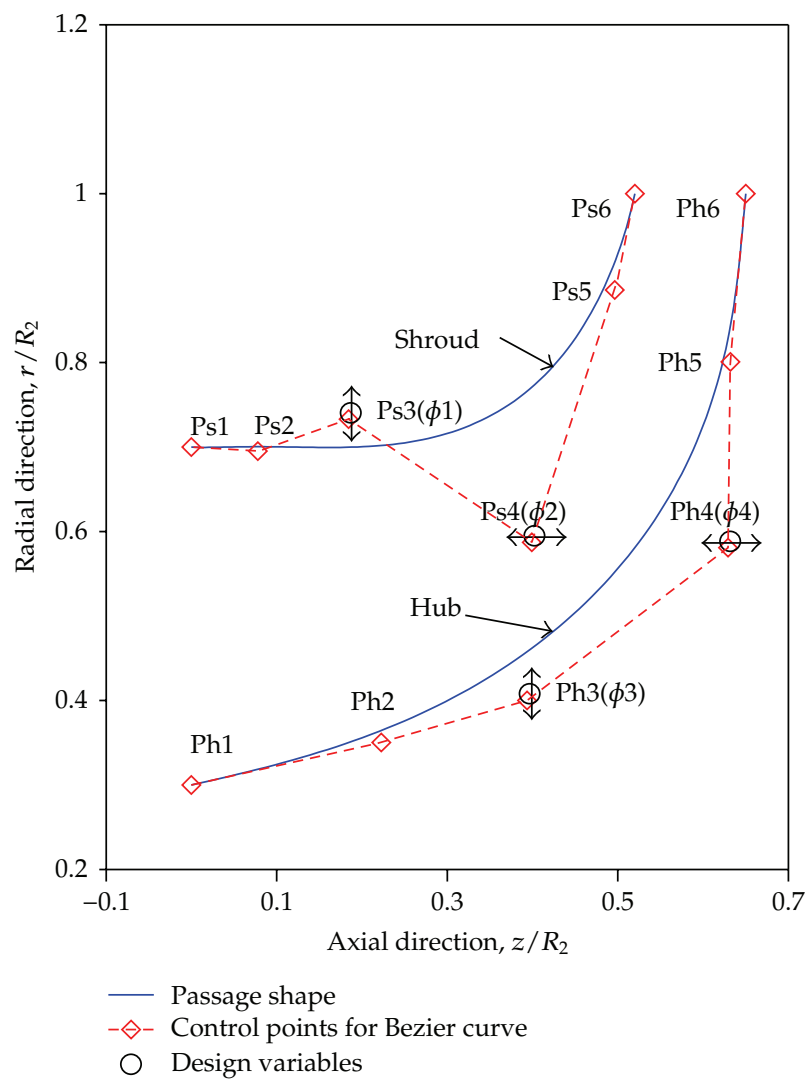

Figure 10: Control points and design variables for the flow passage.

where $\omega$ is the weighting function and the subscripts $\eta$ and $\Pi$ are the efficiency and the pressure ratio, respectively. The objective function $P_{\eta}$ is the difference between the ideal efficiency and the computed total-to-total efficiency. Hence, the maximum efficiency is obtained when the value of $P_{\eta}$ is minimized:

$$
P_{\eta}=1-\eta_{t-t} .
$$




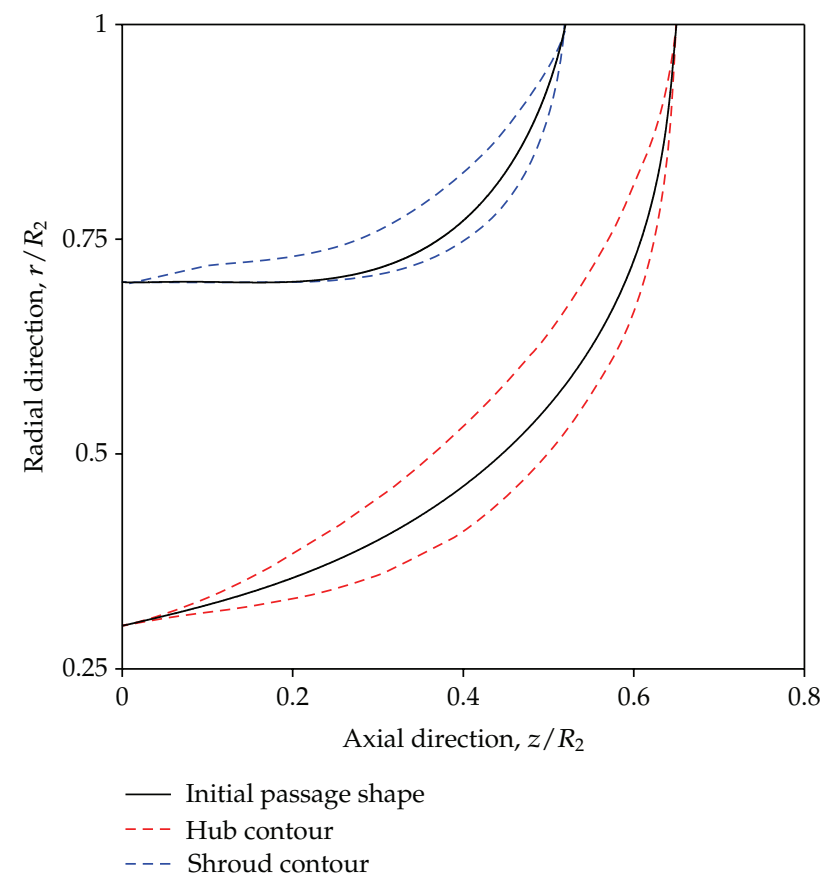

Figure 11: Variation of the passage contour by the design variables.

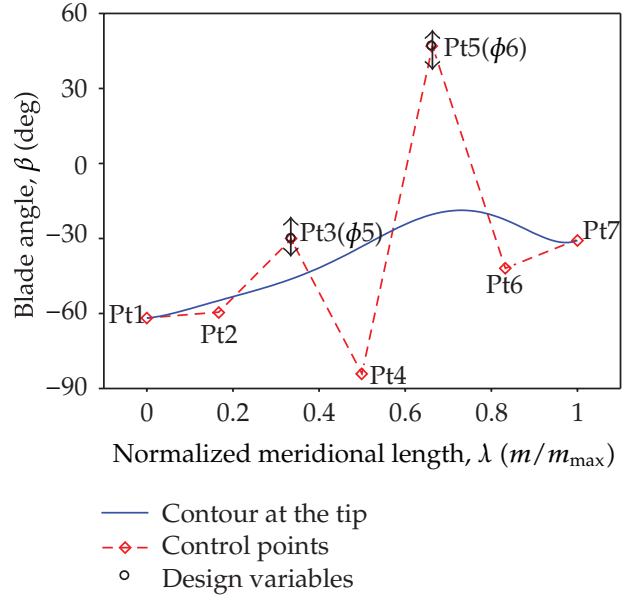

(a)

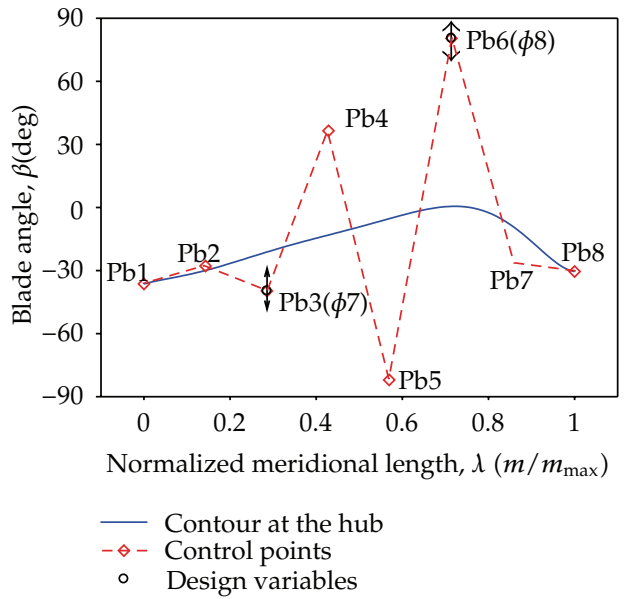

(b)

Figure 12: Control points and design variables for the blade angle contour at the (a) tip and the (b) hub.

In the compressor performance, the pressure ratio is as important a factor as the efficiency. Typically, this pressure ratio is determined in the early design stage depending on the application. If the pressure ratio is lower than the required ratio, the operating pressure should be increased so that the device works properly. In addition, if the pressure ratio is higher than the required ratio, this high pressure causes a loss of power. Therefore, the pressure ratio should be achieved within a very small range based on the requirements. 
In this study, this range was set within $1 \%$ of the necessary pressure ratio $\left(\Pi_{\text {req }}\right)$. If the computed pressure ratio deviates from this range, the objective function of $P_{\Pi}$ is increased. Therefore, the efficiency is maximized while the necessary pressure ratio is compromised, as the objective function (OF) is minimized during the optimization process. In this study, the weighting function was applied equally as one:

$$
P_{\Pi}=\max \left[\left(\frac{\left|\Pi-\Pi_{\text {req }}\right|}{\Pi_{\text {req }}}-0.01\right), 0.0\right] .
$$

When the impeller shape is changed by various design variables during the optimization process, the impeller may be forced to undergo an unacceptable level of mechanical stress. In particular, high mechanical stress arises when the impeller is seriously bent. If this mechanical stress is greater than the allowable stress, the impeller will be unfeasible. Thus, a constraint condition was placed on the mechanical stress as follows:

$$
P_{\sigma}=\frac{\sigma_{\max }-\sigma_{\text {allowable }}}{\sigma_{\text {allowable }}}<0.0 .
$$

\section{Optimization}

\subsection{Optimization Method}

There are many methods of finding the minimum value of the objective function. As a fast method, a gradient-based method can be applied if the objective function rarely reveals nonlinear characteristics as a fan. In this case, the gradient-based method would be a good choice if appropriate initial values are selected so that the optimum does not fall into any local optimum [17]. However, the objective function on the compressor showed numerous local minimums in the design space. If a gradient-based method is applied to this problem, the result could be a local minimum. In order to avoid this undesirable result, a response surface method (RSM) [18, 19] or a genetic algorithm (GA) [20, 21] has been applied, but these methods often require a considerable amount of computational time depending on the number of design variables. To reduce the computational time, a method that combines the RSM and the GA was introduced [10]. In other methods, the ANN has been applied [22,23] and the ANN has been used together with the GA $[8,11,24]$. In addition, the DOE was applied with the RSM and the GA [9].

A trained ANN provides a good methodology to search the global optimum in a problem having numerous local optimums if its accuracy is guaranteed. Hence, the ANN was adopted here as the main method for optimizing the centrifugal compressor showing a nonlinear result. However, the ANN requires much time to be trained, although an optimized result can be obtained quickly from the trained ANN. In order to reduce the training time for building the ANN, the DOE was adopted. As the first step, a design space representing the entire design space was built by a small amount of data that were chosen systematically by the DOE. The initial ANN was then trained and built using the CFD results obtained in this design space. As the second step, new input data from the entire design space were selected using the GA. These input data constitute a generation with its populations. The objective functions for these populations were then evaluated using the initial ANN with the constraints. If any objective function reached a level comparable to the best result obtained in 


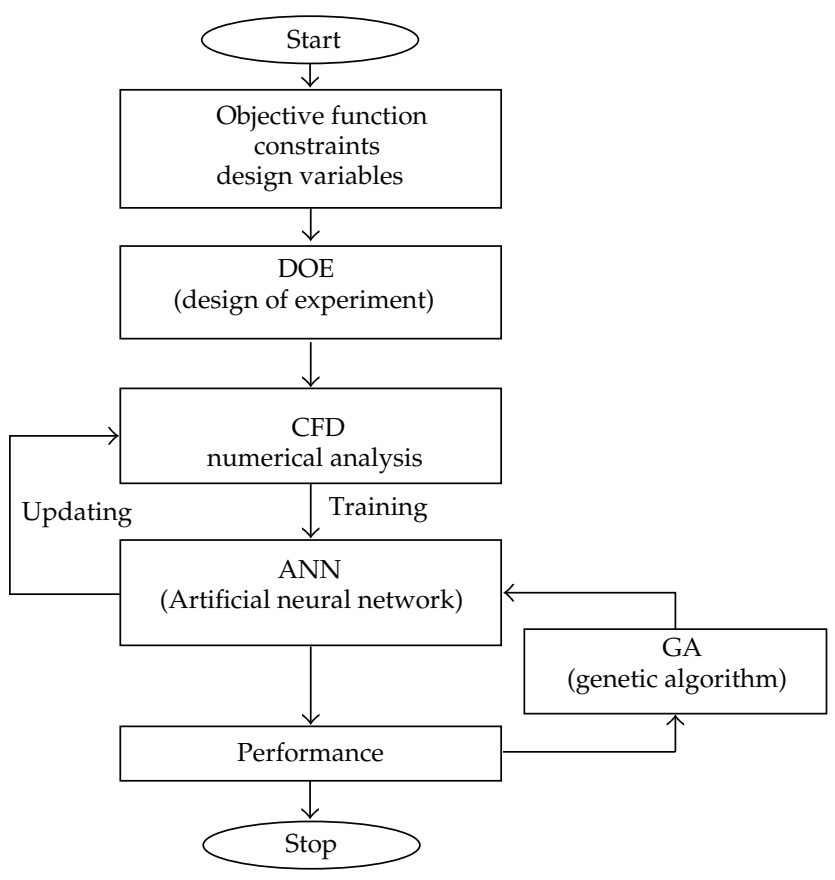

Figure 13: Flow chart of optimization.

the previous step, these results were recalculated using the CFD and were then applied to update the ANN to increase its accuracy. Therefore, the accuracy of the ANN was continuously improved upon each generation of the GA during the optimization process. The working flow for this process is shown in Figure 13.

\subsection{Application}

The fractional factorial design of $2_{V}^{8-2}$ was applied in the DOE for the data set to train the ANN at the beginning. Consequently, the ANN initially required the computed results for sixty-five design cases including a center point. To construct a more accurate ANN, the range of the design variables in the DOE was limited to $75 \%$ of the width from the initial value to the maximum or minimum shown in Table 3 , because the objective function near the minimum and the maximum was worse compared to its initial value. However, during the optimization process, the design variables for new design cases were determined over the entire design space by the GA. For a subsequent generation, the cross-over probability and mutation were chosen as $95 \%$ and $10 \%$, respectively.

For the constraint condition, a stress analysis was conducted using ANSYS version13 for sixty-five design cases. The material of the impeller was chosen as AL7075 alloy. The thickness of the blade was distributed equivalently along the normalized meridional length corresponding with the blade thickness distribution of the original impeller. In the computation, the stress and displacement were calculated under the actual operating conditions. Figure 14 shows the stress distribution on an impeller that is seriously bent among the sixtyfive design cases and the unit of stress is Pascal. The maximum stress resulted at the blade hub located at the exit of the impeller, and the impeller had a safety margin on the stress as 


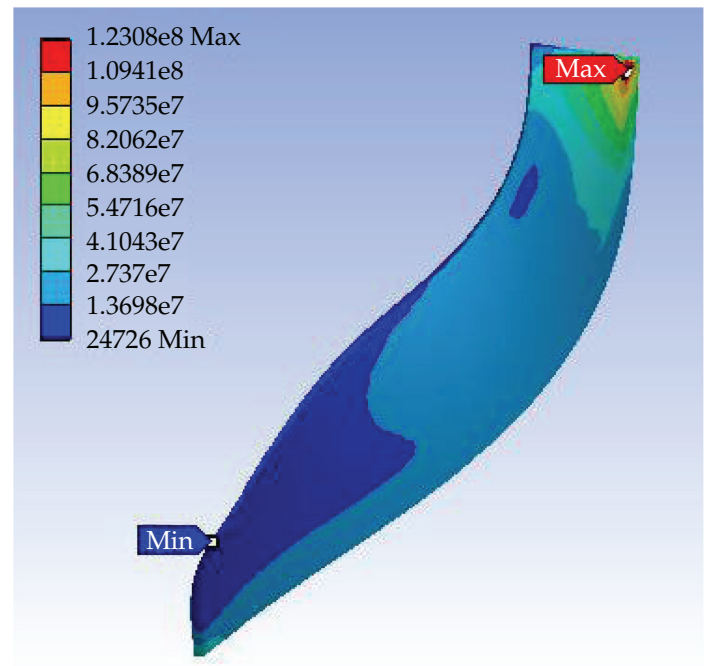

Figure 14: Von Mises stresses due to the centrifugal loading.

well as on the displacement for the sixty-five cases. Therefore, the initial ANN was trained using the results of the sixty-five cases.

A feedforward backpropagation algorithm with two layers was applied to the ANN. A hyperbolic tangent function and a linear function were applied as a transfer function in the hidden layer neuron and a transfer function in the output layer neuron, respectively. Ten neurons were used in the hidden layer. The weights and bias vectors of the networks were searched along the negative gradient of the performance function calculated using the Levernberg-Marquardt algorithm $[25,26]$ with an initial $\mu$ parameter of 0.001 . However, they were updated at a learning rate of 0.1 and a momentum constant of 0.9 so as not to fall in a local minimum while the error, which is the difference between the output predicted by the neural network and the actual output, was decreasing. The training and learning of the network were conducted using the nntool function in MATLAB [27].

From the objective functions for the sixty-five design cases, the main effect or the interaction effect of the design variables was evaluated by the DOE to estimate the influence of each design variable on the objective function. Figure 15 shows the main effect of the design variables. The blade angle on the hub $(\phi 8)$ among the eight design variables shows the strongest influence on the change of the average objective function. However, moving to the $r$-direction on the shroud $(\phi 1)$ weakly affects the change of the average objective function. In the second-order interaction effect of the design variables, the average objective function was mainly influenced by the design variables $(\phi 5, \phi 7),(\phi 7, \phi 8),(\phi 5, \phi 8)$, and $(\phi 2, \phi 4)$. As shown in Figure 16, for the design variables $(\phi 5, \phi 7)$, the interaction effect of the average objective function is increased when the design variable $\phi 5$ is at a high level. For the design variable $(\phi 7, \phi 8)$, the interaction effect is magnified when the design variable $\phi 7$ is at a low level. However, the lowest value of the average objective function was obtained at the center point.

New design cases of one hundred populations upon each generation in the GA were obtained over the entire design space, and their objective functions were then evaluated by the developed ANN. If the output results were less than a designated level on the basis of the minimum obtained in the previous generation, these output results were recalculated using the CFD and were then applied to update the ANN. The value of $\alpha$ in (4.1) was applied as 4 or 


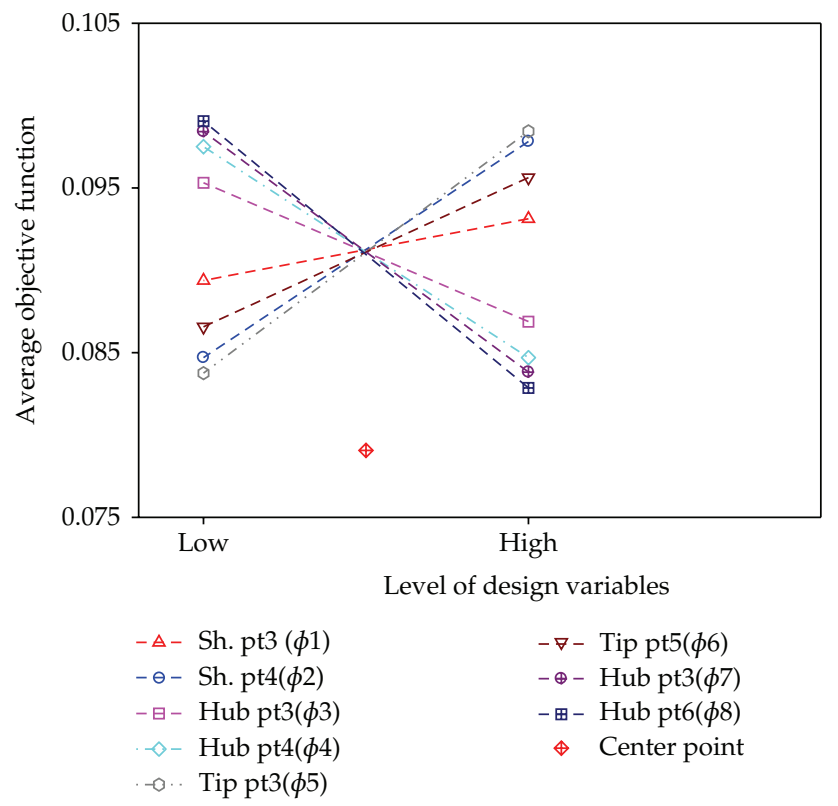

Figure 15: Main effect of the design variables.

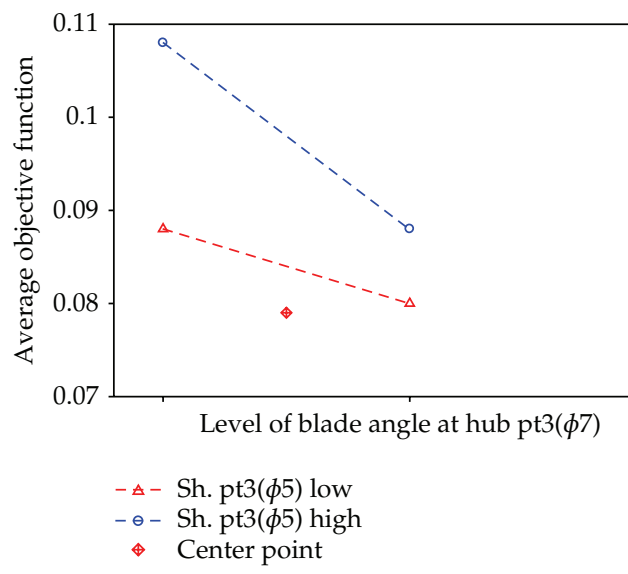

(a)

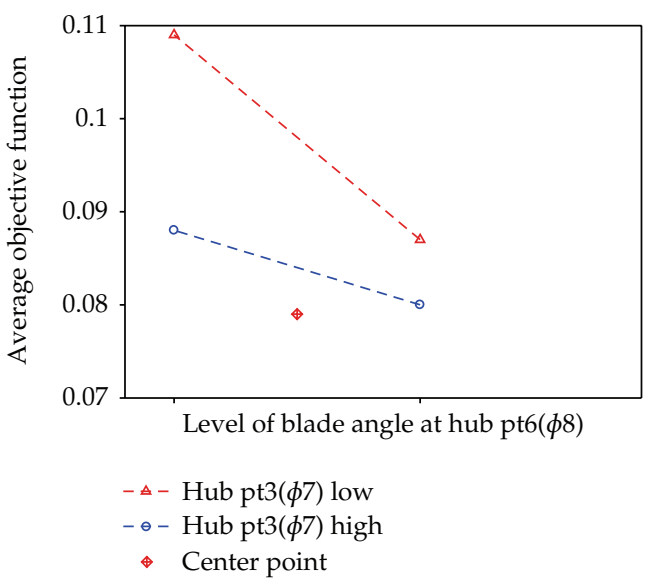

(b)

Figure 16: Interaction effect of the design variables: (a) $\phi 5$ and $\phi 7$ and (b) $\phi 7$ and $\phi 8$.

$5 \%$ depending on the generation. Table 4 shows that this ANN was continuously improved upon advancing to a new generation. The error, which is the average difference between the outputs predicted by the ANN and those by the CFD, was decreased to less than $1 \%$ upon the sixth generation:

$$
\mathrm{OF}_{\text {new }} \leq \mathrm{OF}_{\min (\text { old })}+\alpha \times \mathrm{OF}_{\min (\text { old })}
$$


Table 4: Number of updated design variables and errors.

\begin{tabular}{lccc}
\hline Generations & Updated design variables & Error [\%] & Level $(\alpha)[\%]$ \\
\hline 1st & 12 & 8.23 & 5.0 \\
2nd & 9 & 3.50 & 5.0 \\
3rd & 14 & 3.67 & 5.0 \\
4th & 8 & 1.57 & 5.0 \\
5th & 11 & 1.60 & 4.0 \\
6th & 11 & 0.92 & 4.0 \\
\hline
\end{tabular}

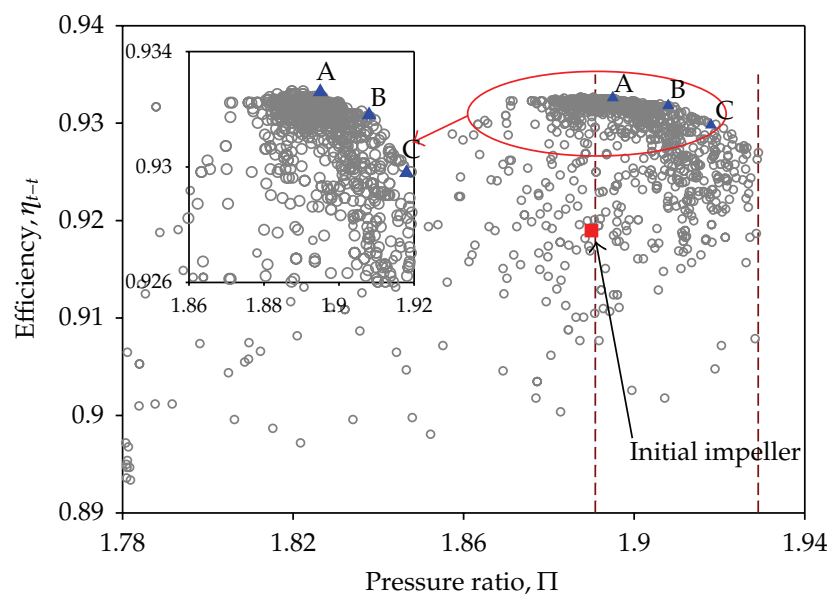

Figure 17: Paretooptimal solutions of the objective function.

After advancing to the twenty-first generation, the objective function could not decrease further. Figure 17 shows the efficiency and pressure ratio for all design cases. The optimum of the objective function defined as (3.1) is indicated by " $\mathrm{A}$ " in Figure 17. The efficiency was improved by $1.4 \%$ compared to the initial compressor without penalizing the pressure ratio. In addition, Figure 17 shows the Paretooptimal solutions of the efficiency versus the pressure ratio. The efficiency on the Paretooptimal front $(\mathrm{POF})$ increases when the pressure ratio decreases; otherwise, the pressure ratio increases as the efficiency decreases. Hence, any location on the POF can be selected as the design point depending on the operating conditions. Table 5 shows the design variables in the initial conditions as well as in the operating conditions on the POF.

Total pressure contours obtained upon the initial configuration were compared with those obtained at the operating points " $\mathrm{A}$ ", " $\mathrm{B}$ ", and " $\mathrm{C}$ " on the POF. Figure 18 shows how the shape of the flow passage has been modified from the initial configuration depending on the operating points, because they are illustrated in the meridian plane. When the operating point is moved to " $\mathrm{C}$ " from " $\mathrm{A}$ ", the pressure ratio is gradually increased and the shape of the flow passage is gradually altered, as shown in Figure 18. In particular, the curvature of the shroud is decreased according to the increase in the pressure ratio. This reduced curvature increases the height of the flow passage near the exit of the impeller, which causes an increase in the pressure ratio.

Relative Mach contours in the meridian plane are shown in Figure 19. A low-velocity region is generated near the hub at the operating point " $\mathrm{C}$ ". This low-velocity region is 
Table 5: Comparison of the design variables located on the Paretooptimal front.

\begin{tabular}{lcccc}
\hline Design variables & Initial & $\mathrm{A}$ & $\mathrm{B}$ & $\mathrm{C}$ \\
\hline$\phi 1, \mathrm{Ps} 3\left[r / R_{2}\right]$ & 0.733 & 0.779 & 0.753 & 0.790 \\
$\phi 2, \mathrm{Ps} 4\left[z / R_{2}\right]$ & 0.399 & 0.345 & 0.279 & 0.266 \\
$\phi 3, \mathrm{Ph} 3\left[r / R_{2}\right]$ & 0.400 & 0.518 & 0.518 & 0.473 \\
$\phi 4, \mathrm{Ph} 4\left[z / R_{2}\right]$ & 0.629 & 0.638 & 0.678 & 0.678 \\
$\phi 5, \mathrm{Pt} 3[$ angle] & -30.022 & -19.746 & -20.548 & -20.548 \\
$\phi 6, \mathrm{Pt} 5$ angle] & 46.949 & 20.414 & 29.037 & 33.249 \\
$\phi 7, \mathrm{~Pb} 3$ [angle] & -39.754 & -31.550 & -33.556 & -33.556 \\
$\phi 8, \mathrm{~Pb} 6$ [angle] & 80.409 & 86.899 & 79.679 & 80.682 \\
\hline
\end{tabular}

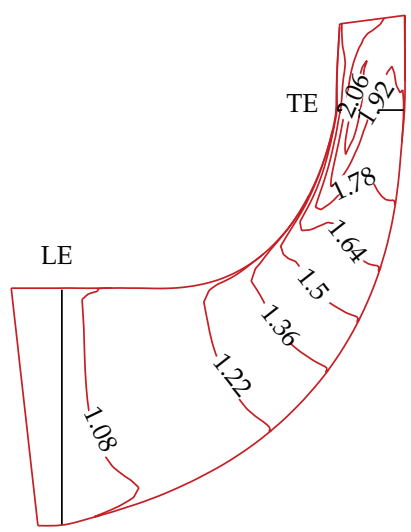

(a)

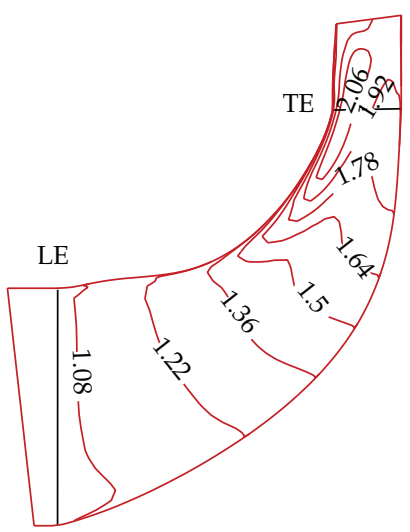

(b)

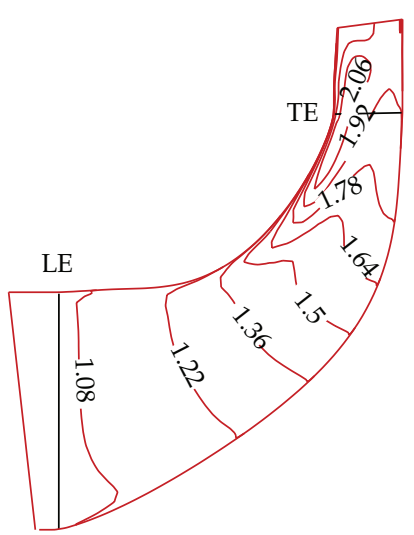

(c)

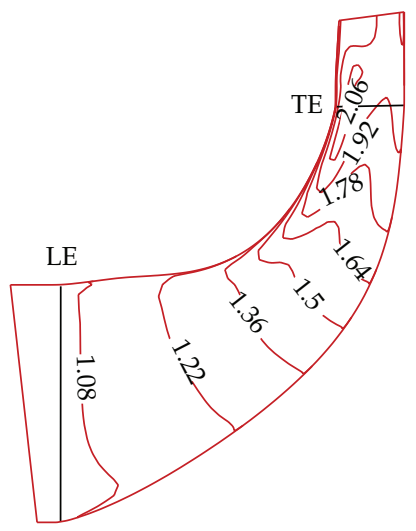

(d)

Figure 18: Comparison of total pressure contours at (a) initial, (b) " $C^{\prime \prime},(c)$ " $\mathrm{B}^{\prime}$, and (d) " $\mathrm{A}$ ".

gradually reduced when the operating condition moves to the operating point of the lowpressure ratio. The relative velocity at the exit of the impeller becomes slower when the compressor operates at the POF compared to the initial state. Figure 20 shows the relative velocities obtained along the blade-to-blade at the midspan where is located on the surface aligned normal to the meridional shroud contour. At the operating point " $\mathrm{C}$ ", the relative velocities are slightly slower than those obtained at other operating points. In particular, the relative velocities obtained at the POF have a profile that differs from those of the initial 


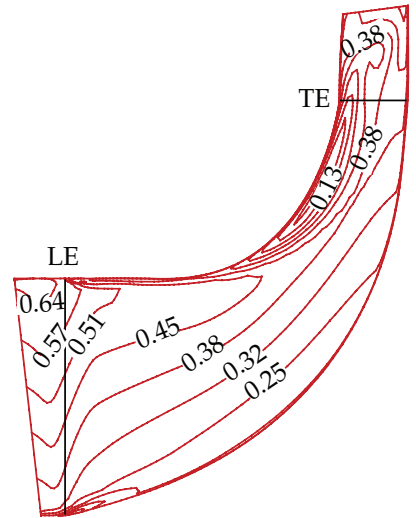

(a)

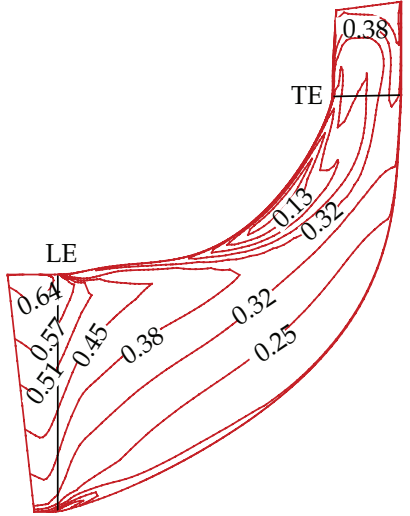

(b)

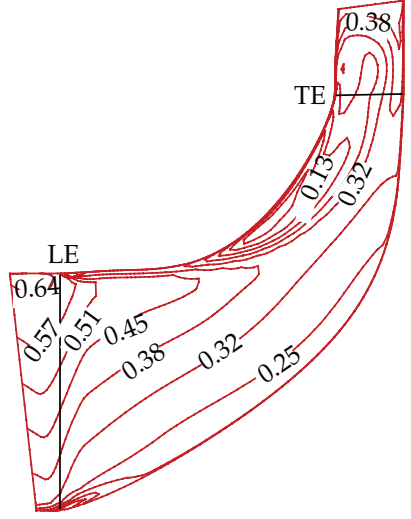

(c)

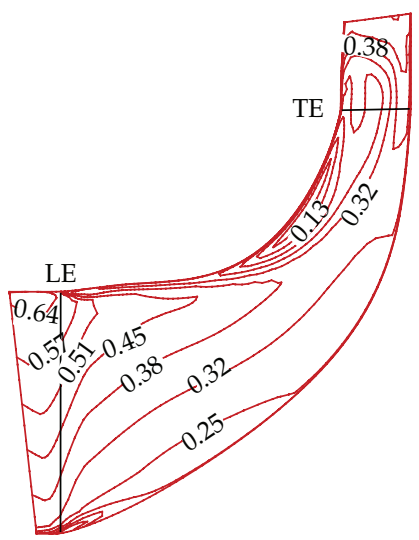

(d)

Figure 19: Comparison of relative Mach number contours at (a) initial, (b) " $C$ ", (c) " $B$ ", and (d) " $A$ ".

configuration at the half pitch near the exit of the impeller due to the change in the shroud contour.

\section{Conclusions}

An optimization study was conducted on a centrifugal compressor of which the shape was expressed by the data of numerous points. This point data set was converted to Bezier curves to determine the best design variables. Each shape for the impeller blade or the flow passage could be fully expressed by six to eight control points for each Bezier curve. Among these control points, eight control points that strongly affect the change of the shape were chosen as design variables. The objective function was considered in terms of both the efficiency and the pressure ratio, and the limitation of the mechanical stress was set as a constraint. For the optimization algorithm, the ANN was adopted and it was trained and updated to improve its accuracy using the DOE with a GA. After advancing to the sixth generation, the prediction error between the ANN and the CFD was reduced to less than $1 \%$. The Paretooptimal solutions were obtained after the twenty-first generation. The efficiency was improved by $1.4 \%$ without loss of the pressure ratio. 


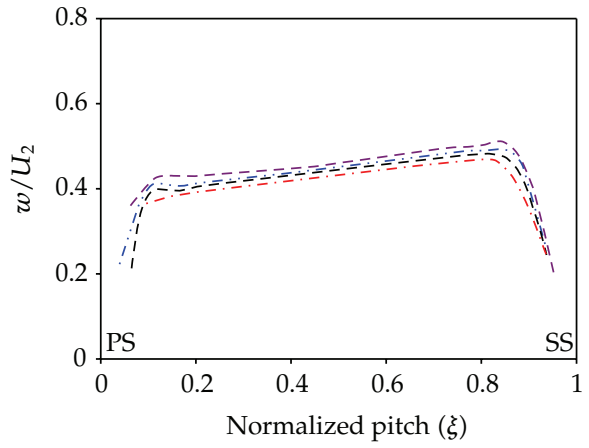

(a)

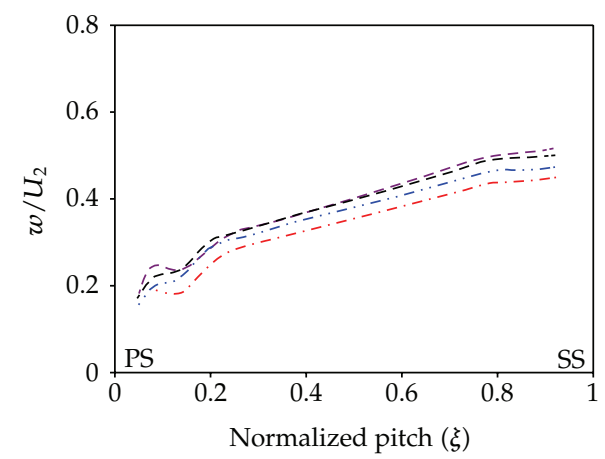

(c)

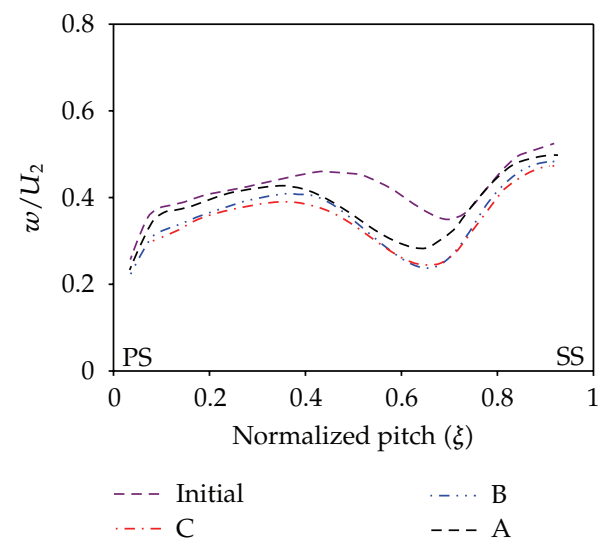

(e)

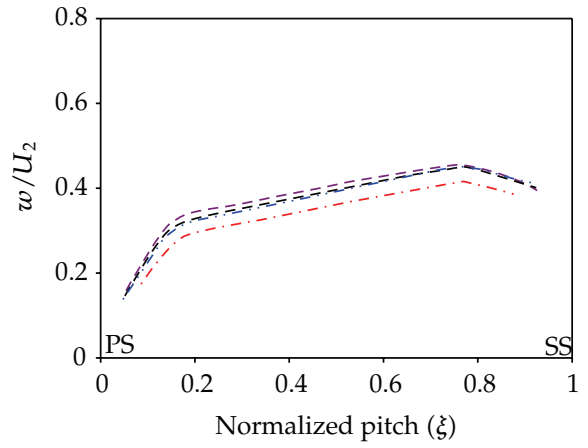

(b)

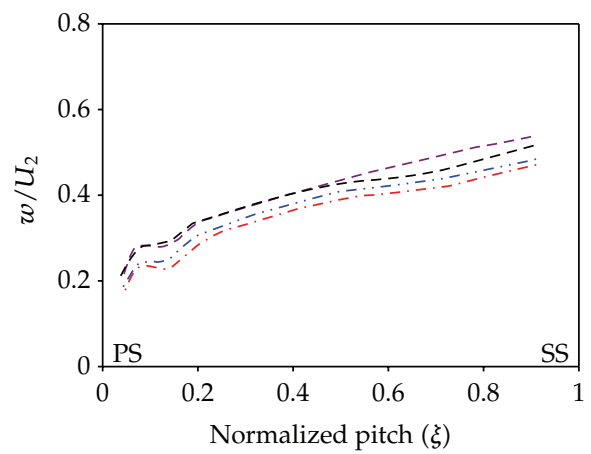

(d)

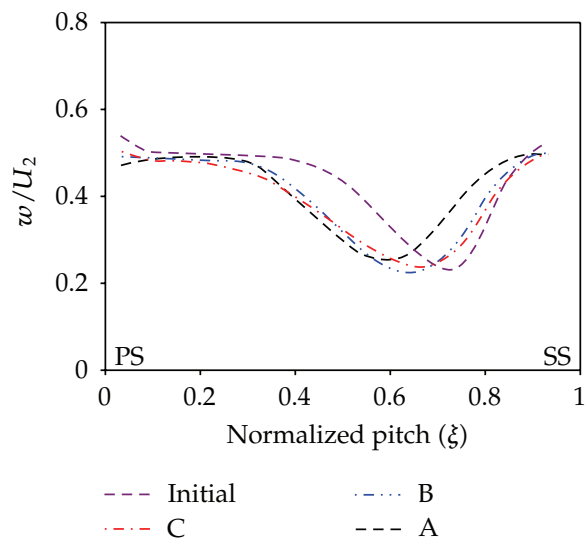

(f)

Figure 20: Relative velocities along the blade-to-blade at the mid span: (a) $\lambda=0.08$, (b) $\lambda=0.43$, (c) $\lambda=0.59$, (d) $\lambda=0.73$, (e) $\lambda=0.87$, and (f) $\lambda=1.01$.

\section{Acknowledgments}

This work was supported by the Degree and Research Center for Aerospace Green Technology (DRC) of the Korea Aerospace Research Institute (KARI) funded by the Korea Research Council of Fundamental Science \& Technology (KRCF) and by the turbocharger development project by the Korea Institute of Machinery and Materials (KIMM). 


\section{References}

[1] A. R. Forrest, "Interactive interpolation and approximation by bézier polynomials," Computer Journal, vol. 15, no. 1, pp. 71-79, 1972.

[2] F. J. Wallace, A. Whitfield, and R. Atkey, "A computer-aided design procedure for radial and mixed flow compressors," Computer-Aided Design, vol. 7, no. 3, pp. 163-170, 1975.

[3] M. V. Casey, "A computational geometry for the blades and internal flow channels of centrifugal compressors," Journal of Engineering for Power, vol. 105, no. 2, pp. 288-295, 1983.

[4] N. A. Cumpsty, Compressor Aerodynamics, Longman Group UK, 1989.

[5] A. Whitfield and N. C. Baines, Design of Radial Turbomachines, Longman Group UK, 1990.

[6] R. H. Aungier, Centrifugal Compressor, ASME Press, 2000.

[7] D. Bonaiuti and V. Pediroda, "Aerodynamic optimization of an industrial centrifugal compressor impeller using genetic algorithms," in Proceedings of Eurogen, 2001.

[8] R. Cosentino, Z. Alsalihi, and V. R. A. Braembussche, "Expert system for radial impeller optimization," in Proceedings of Euroturbo, 2001.

[9] D. Bonaiuti, A. Arnone, M. Ermini, and L. Baldassarre, "Analysis and optimization of transonic centrifugal compressor impellers using the design of experimental technique," GT-2002-30619, 2002.

[10] D. Bonaiuti and M. Zangeneh, "On the coupling of inverse design and optimization techniques for the multiobjective, multipoint design of turbomachinery blades," Journal of Turbomachinery, vol. 131, no. 2, Article ID 021014, 16 pages, 2009.

[11] T. Verstraete, Z. Alsalihi, and R. A. Van den, "Multidisciplinary optimization of a radial compressor for microgas turbine applications," Journal of Turbomachinery, vol. 132, no. 3, Article ID 031004, 7 pages, 2010.

[12] D. Eckardt, "Instantaneous measurements in the jet-wake discharge flow of a centrifugal compressor impeller," Journal of Engineering and Power, vol. 97, no. 3, pp. 337-346, 1975.

[13] D. Eckardt, "Detailed flow investigations within a high-speed centrifugal compressor impeller," Journal of Fluid Engineering, vol. 98, no. 3, pp. 390-402, 1976.

[14] D. Eckardt, "Flowfield analysis of radial and backswept centrifugal compressor impellers-part 1: flow measurements using a laser velocimeter," in Performance Prediction of Centrifugal Pumps and Compressor, S. Gopalskrishnan and P. Cooper, Eds., ASME, 1980.

[15] CFX-12, version 12, Ansys Inc, 2009.

[16] P. Schuster and U. Schmidt-Eisenlohr, "Flowfield analysis of radial and backswept centrifugal compressor impellers-part 2: comparison of potential flow calculations and measurements," in Performance Prediction of Centrifugal Pumps and Compressor, S. Gopalskrishnan and P. Cooper, Eds., ASME, 1980.

[17] C. H. Cho, S. Y. Cho, K. Y. Ahn, and Y. C. Kim, "Study of an axial-type fan design technique using an optimization method," Proceedings IMechE Journal of Process Mechanical Engineering, vol. 223, no. 3, pp. 101-111, 2009.

[18] Z. Wang, G. Xi, and X. Wang, "Aerodynamics design optimization of vaned diffuser for centrifugal compressors using Kriging model," in Proceeding of Asian Joint Conference on Propulsion and Power (AJCPP '06), Beijing, China, 2006.

[19] X. Shu, C. Gu, J. Xiao, and C. Gao, "Centrifugal compressor blade optimization based on uniform design and genetic algorithms," Frontiers of Energy and Power Engineering in China, vol. 2, no. 4, pp. 453-456, 2008.

[20] H. Y. Fan, "An inverse design method of diffuser blades by genetic algorithms," Proceedings IMechE Journal of Power and Energy, vol. 212, no. 4, pp. 261-268, 1998.

[21] E. Benini, “Optimal Navier-Stokes design of Ccompressor impellers using evolutionary computation," International Journal of Computational Fluid Dynamics, vol. 17, no. 5, pp. 357-369, 2003.

[22] S. Pierret and R. A. Van Den Braembussche, "Turbomachinery blade design using a Navier-Stokes solver and Artificial Neural Network," Journal of Turbomachinery, vol. 121, no. 2, pp. 326-332, 1999.

[23] H. Y. Fan, "A neural network approach for centrifugal impeller inverse design," Proceedings IMechE Journal of Power and Energy, vol. 214, no. 2, pp. 183-186, 2000.

[24] J. H. Kim, J. H. Choi, A. Husain, and K. Y. Kim, "Multi-objective optimization of a centrifugal compressor impeller through evolutionary algorithms," Proceedings IMechE Journal of Power and Energy, vol. 224, no. 5, pp. 711-721, 2010. 
[25] M. T. Hagan and M. B. Menhaj, "Training feedforward networks with the Marquardt algorithm," IEEE Transactions on Neural Networks, vol. 5, no. 6, pp. 989-993, 1994.

[26] M. T. Hagan, H. B. Demuth, and M. H. Beale, Neural Network Design, PWS Publishing, Boston, Mass, USA, 1996.

[27] H. Demuth, M. Beale, and M. Haga, “Neural Network Toolbox 6, User’s Guide," Matlab R2007b, 2007. 


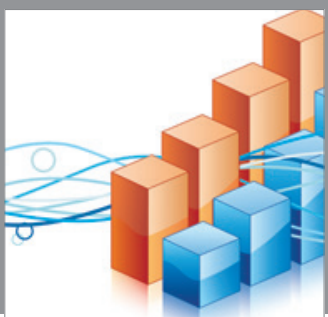

Advances in

Operations Research

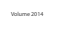

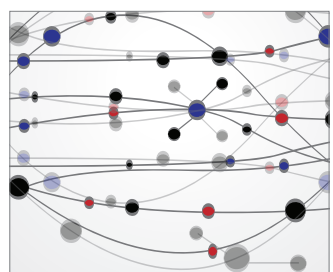

\section{The Scientific} World Journal
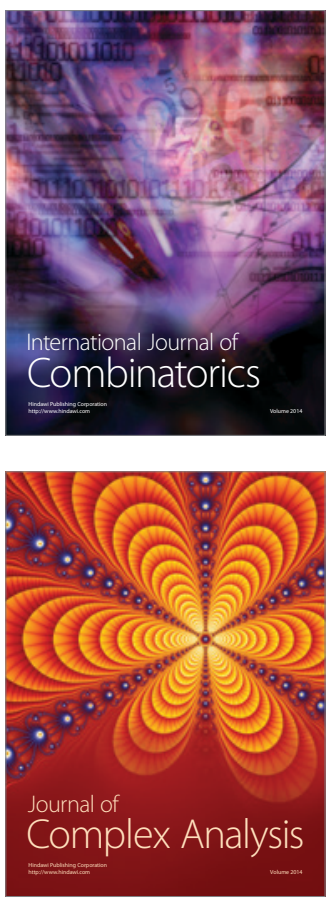

International Journal of

Mathematics and

Mathematical

Sciences
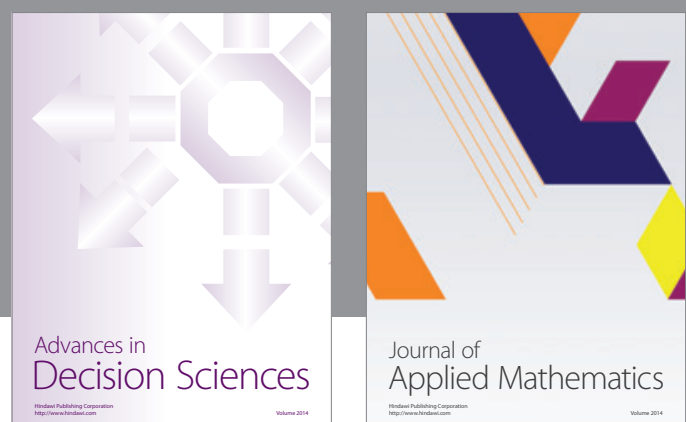

Journal of

Applied Mathematics
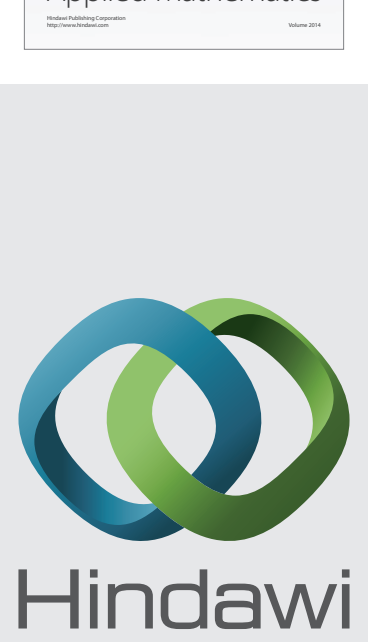

Submit your manuscripts at http://www.hindawi.com
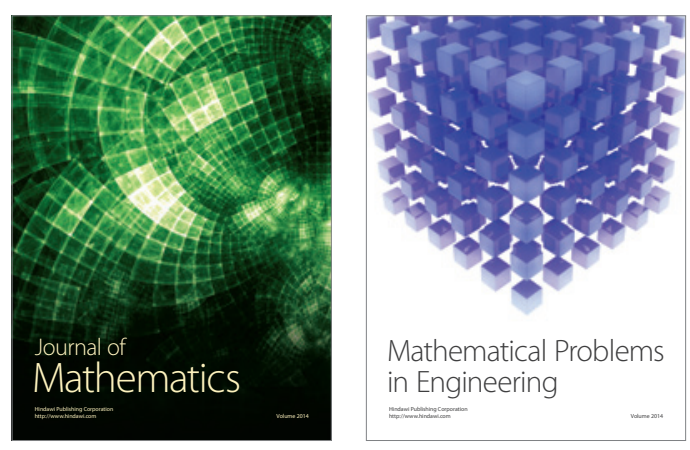

Mathematical Problems in Engineering
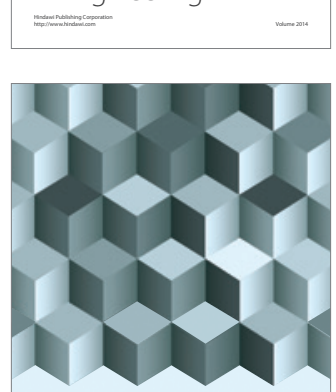

Journal of

Function Spaces
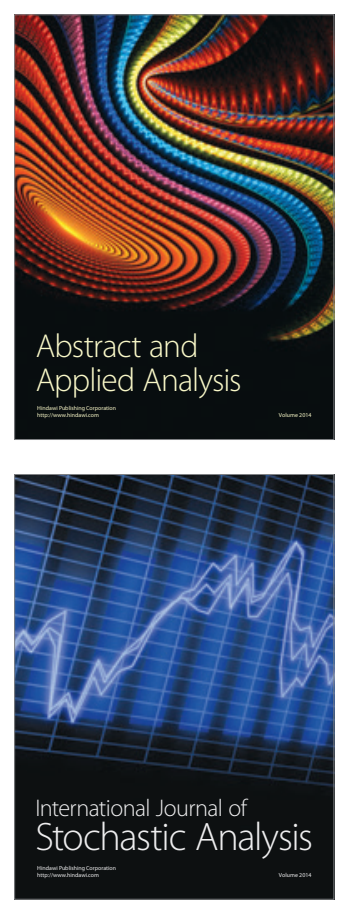

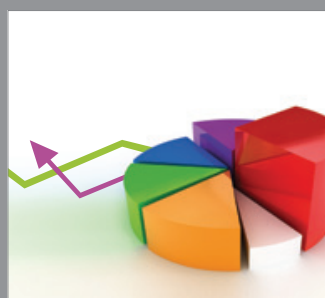

ournal of

Probability and Statistics

Promensencen
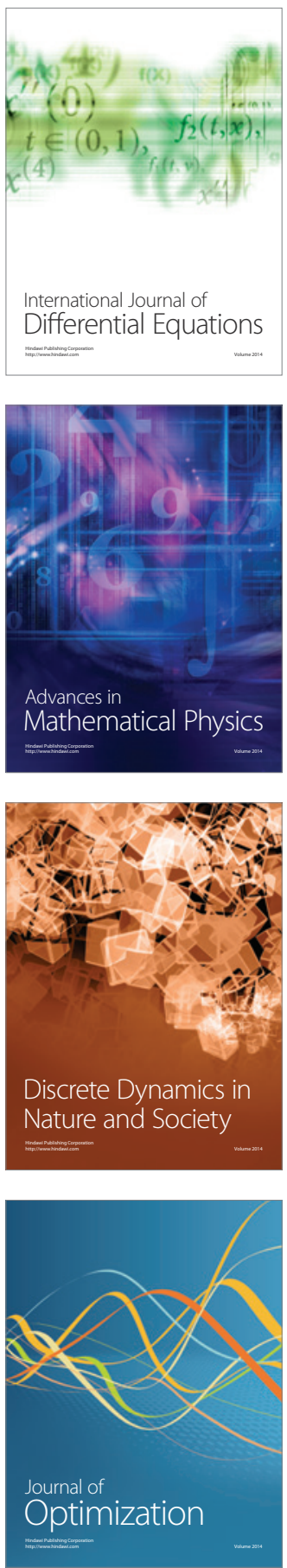